\title{
La importancia de las empresas de servicios en el desarrollo de los países de economías emergentes
}

\section{The importance of service companies in the development of emerging market countries}

María Auxiliadora Guerrero Bejarano, MBA

Universidad Internacional del Ecuador, Ecuador

Autor para correspondencia: maguerrerobe@uide.edu.ec

Fecha de recepción: 08 de Octubre 2017 - Fecha de aceptación: 15 de Febrero de 2018

\section{Resumen}

Este análisis teórico tiene como objetivo resaltar la importancia que tienen las empresas de servicio en el desarrollo de las economías emergentes, entendiendo que el Ecuador es un país que se clasifica dentro de este tipo de economías y en el que se encuentra un alto número de emprendimientos generalmente establecidos a partir de la necesidad de los ciudadanos de generar nuevas formas de ingresos. Se encuentra que en el país más del $40 \%$ de las empresas son del sector de servicios y su producción aporta cerca del $60 \%$ del PIB.

Palabras Clave: economía emergente; servicios; desarrollo

\begin{abstract}
This theoretical analysis aims to highlight the importance of service companies in the development of emerging economies, understanding that Ecuador is a country that is classified within this type of economy and in which there is a high number of ventures generally established from the need of citizens to generate new forms of income. It is found that in the country more than $40 \%$ of the companies are from the service sector and their production contributes about $60 \%$ of GDP.
\end{abstract}

Key words: emergent economies; services; development 


\section{Introducción}

Desde hace décadas los países de las diferentes regiones del mundo, han venido atravesando crisis financieras que obligan a las empresas y personas a buscar vías creativas de desarrollo para enfrentar las carencias que dichas crisis ocasiones; en algunos casos estas situaciones son consecuencia de la globalización y su efecto a nivel mundial; siendo la mayoría consecuencia de las políticas gubernamentales que afectan la forma en la que se desarrollan las empresas en cada país.

En Latinoamérica, casi todos los países son considerados de economía emergente, y se aprecia cómo las poblaciones buscan afrontar situaciones apremiantes a partir de la generación de nuevos negocios. A pesar de no contar con grandes capitales o acceso a crédito, en la mayoría de los casos, se puede apreciar el surgimiento de pequeños negocios que se caracterizan por ofrecer intangibles o productos tangibles enmarcados en políticas de servicio que ofrecen valores agregados a sus clientes aprovechando las nuevas tecnologías y las nuevas formas de consumo(Gémar Castillo \& Jiménez Quintero, 2013; Sánchez-Bayon, Lafuente, \& de Quevedo, 2018).

\section{Empresas de Servicios}

Las empresas de servicios son aquellas que se dedican como actividad principal a la satisfacción de las necesidades de los clientes a través de la venta de intangibles, existiendo una amplia variedad de empresas dentro de este sector. Desde hace décadas han ido ganando mayor espacio dentro del desarrollo de las empresas en los países, siendo en algunos casos las fuentes principales de ingresos y desarrollo de éstos (Edvardsson, 1992; Gallarza \& Gil, 2006).

El consumo de servicios en los países se ha convertido en un indicador del nivel de vida de los ciudadanos (Rubalcaba Bermejo, Gago, Ariano, \& Tripathi, 2016), como casi todas las industrias, la de servicio se encuentra en constante cambio debido a la tecnología y su influencia en la forma de hacer las cosas (Descals, Eiglier, \& Langeard, 1989; Lovelock, Carrion, Huete, Reynoso, \& D'Andrea, 2004), lo que permite a pequeñas empresas competir con empresas más grandes. De la misma manera las empresas productoras de bienes, encuentran en la prestación de servicios post venta una fuente de ingresos que en algunas ocasiones consiguen constituir un porcentaje significativo en el total de los ingresos de dichas empresas (De Gómez \& De Berrios, 2008; Mera, del Rosario, Lara Burbano, Carrillo, \& Maribel, 2018).

\section{Economías Emergentes}

Los países denominados de economías emergentes se caracterizan por en primer lugar tener gran déficit fiscales, normalmente financiados desde el exterior, los sistemas financieros por otro lado, suelen encontrarse reprimidos lo que también afecta a la accesibilidad de créditos para las empresas del sector privado. Otra característica frecuente es la crisis presente en la balanza de pagos que suele asociarse a los efectos de las decisiones políticas que afectan el intercambio comercial desde y hacia el exterior. Adicionalmente en este tipo de economías se encuentran niveles de desempleos altos, así como tasas de subempleo y empleos informales más altas (de Mattos, 1998, 2001; Ffrench-Davis \& Ocampo, 2001). 
Ecuador cumple con todas estas características, lo que ha generado que se convierta en uno de los países de Latinoamérica con mayores índices de emprendimiento, de acuerdo con los índices de los años 2009 al 2014 (Mera et al., 2018). En un estudio realizado que analiza la actividad emprendedora y competitividad en el país, se encontró que los emprendimientos son motivados por la necesidad con pequeños cambios hacia la oportunidad; el índice de competitividad en el Ecuador ha ido escalando posiciones aunque se mantiene como una economía más enfocada en la eficiencia y no en la innovación (Mera et al., 2018).

En el Ecuador el 40,8\% de las empresas son de servicios; a este porcentaje podría sumarse el 36,5\% de empresas que se dedican al comercio, donde el servicio es parte integral de su actividad (INEC, 2014), de acuerdo con un reportaje del Banco Mundial, uno de los problemas que el sector servicios enfrenta en el país es la baja productividad; contribuyendo menos al PIB que el mismo sector en otros países de Latinoamérica (Rubalcaba Bermejo et al., 2016); estos problemas afectan de forma negativa a la economía del país (Daude \& FernándezArias, 2010).

De acuerdo con datos oficiales, en el Ecuador, la balanza de servicios ha sido tradicionalmente negativa, en el 2014 en 1220 millones de dólares. Aún no existen suficientes estadísticas de este sector por lo que aún se considera un sector desconocido, pese a aportar el 60\% del PIN (Falconí, 2015). En la actualidad este sector genera 1,9 millones de empleos en el país (Telégrafo, 2015) y las empresas de servicio representan el 40,8 \% del total de las empresas del país (INEC, 2014).

De acuerdo con Falconí, coordinador técnico de negociaciones comerciales del ministerio de comercio exterior del Ecuador, la ineficiencia de los servicios en Ecuador no permite que el país aproveche ventajas de acuerdos comerciales, lo que postergaría el futuro del país en un mundo interrelacionado (2015). De acuerdo a un estudio previo realizado por el instituto de estudios económicos de la Universidad Técnica Particular de Loja, existiría un desarrollo del sector en el país, pero con baja productividad presente en el mismo (Ordoñez, 2011).

\section{Conclusiones}

A partir de este análisis se puede concluir que las organizaciones del sector de servicios cobran más importancia en las economías de los países, pero con mayor fuerza en los de economías emergentes, debido al desarrollo de pequeños negocios que nacen dentro del sector y que en algunos casos sobreviven en el tiempo (Kantis, Ishida, \& Komori, 2002). En el Ecuador, la realidad no distal del resto de países de Latinoamérica, aunque tiene sus características como que un alto porcentaje de los emprendimientos están vinculados con el sector del turismo (Turismo, 2014). Se puede apreciar un crecimiento en el sector de servicios tecnológicos también, o en la implementación de servicios a través de plataformas tecnológicas (Mera et al., 2018).

En países como el Ecuador, dónde el aparato estatal es una de las fuentes más significativas de empleo y es el responsable de la prestación de la mayoría de los servicios para la población, el servicio cobra mayor importancia, razón por la que se esperaría se empezara a 
realizar más estudios sobre empresas de este sector en el país, que permitan conocer de mejor forma su rendimiento y la afectación de estas en la economía de la nación (Comesaña, 2007).

\section{Bibliografía}

Comesaña, A. C. (2007). De consumidor a ciudadano: el papel de la satisfacción del ciudadano en la sostenibilidad de los mercados de servicios públicos. Información comercial española.

Daude, C., \& Fernández-Arias, E. (2010). On the role of productivity and factor accumulation in economic development in Latin America and the Caribbean.

De Gómez, M. Y. B., \& De Berrios, O. G. (2008). La servucción y la calidad en la fabricación del servicio. Visión gerencial(1), 21-32.

de Mattos, C. A. (1998). Reestructuración, crecimiento y expansión metropolitana en las economías emergentes latinoamericanas. Economía Sociedad y Territorio.

De Mattos, C. A. (2001). Movimientos del capital y expansión metropolitana en las economías emergentes latinoamericanas. Revista de estudios regionales(60).

Descals, A. M., Eiglier, P., \& Langeard, E. (1989). Servucción: McGraw Hill.

Edvardsson, B. (1992). Service breakdowns: a study of critical incidents in an airline. International Journal of Service Industry Management, 3(4), 17-29.

Falconí, J. (2015). Los Servicios en el Ecuador: Algunos desafios a Corto Plazo. Paper presented at the United Nations Conference on Trade an Development, Geneva. http://unctad.org/meetings/en/Presentation/c1mem3_2015_p18_Falconi_en.pdf

Ffrench-Davis, R., \& Ocampo, J. A. (2001). Globalización de la volatilidad financiera: desafíos para las economías emergentes. En: Crisis financieras en países' exitosos'-Santiago: McGraw-Hill Interamericana/CEPAL, 2001-p. 1-41.

Gallarza, M. G., \& Gil, I. (2006). Desarrollo de una escala multidimensional para medir el valor percibido de una experiencia de servicio. Revista Española de Investigación de marketing ESIC, 18, 35-60.

Gémar Castillo, G., \& Jiménez Quintero, J. A. (2013). Retos estratégicos de la industria hotelera española del siglo xxi: horizonte 2020 en países emergentes. Tourism \& Management Studies, 9(2).

INEC. (2014). Directorio de Empresas y Establecimientos. Retrieved from http://www.ecuadorencifras.gob.ec/documentos/webinec/Estadisticas_Economicas/DirectorioEmpresas/Empresas_2014/Principales_Resultad os_DIEE_2014.pdf 
Kantis, H., Ishida, M., \& Komori, M. (2002). Empresarialidad en economías emergentes: Creación y desarrollo de nuevas empresas en América Latina y el Este de Asia. Retrieved from

Lovelock, C. H., Carrion, M. A. S., Huete, L. M., Reynoso, J., \& D'Andrea, G. (2004). Administración de servicios: Pearson Education.

Mera, M., del Rosario, B., Lara Burbano, G. J., Carrillo, M., \& Maribel, A. (2018). Actividad Emprendedora Y Competitividad En El Ecuador (Entrepreneurship and Competitiveness in Ecuador).

Ordoñez, J. A. (2011). Informe de Coyntura Económica $N^{o} 7$. Retrieved from http://www.utpl.edu.ec/comunicacion/wp-content/uploads/2012/12/utpl-Informe-decoyuntura-economica-N-7-ano-2011.pdf

Rubalcaba Bermejo, L., Gago, D., Ariano, M., \& Tripathi, A. K. (2016). Services and Innovation for the Competitiveness of the Ecuadorian Economy.

Sánchez-Bayon, A., Lafuente, C. F., \& de Quevedo, G. C. G. (2018). Plan de acción frente al consumismo global de la Nueva Economía: Revelaciones sobre economía, empresa y consumo del s. XXI. Revista Empresa y Humanismo, 21(1), 69-93.

Telégrafo, E. (2015). El sector servicios genera 1,9 millones de empleos. Retrieved from http://www.eltelegrafo.com.ec/noticias/economia/1/el-sector-servicios-genera-19millones-de-empleos

Turismo, M. d. (2014). Estadísticas Turísticas. Retrieved from http://servicios.turismo.gob.ec/index.php/anuario-de-estadisticas-turisticas 